\title{
Validade convergente do Inventário de Empatia (IE)
}

\author{
Eliane Mary de Oliveira Falcone - Universidade do Estado do Rio de Janeiro, Rio de Janeiro, Brasil \\ Vanessa Dordron de Pinho - Universidade do Estado do Rio de Janeiro, Rio de Janeiro, Brasil \\ Maria Cristina Ferreira - Universidade Salgado de Oliveira, Rio de Janeiro, Brasil \\ Conceição dos Santos Fernandes - Universidade do Estado do Rio de Janeiro, Rio de Janeiro, Brasil \\ Juliana Furtado D'Augustin - Universidade do Estado do Rio de Janeiro, Rio de Janeiro, Brasil \\ Stèphanie Krieger - Universidade do Estado do Rio de Janeiro, Rio de Janeiro, Brasil \\ Monique Gomes Plácido - Universidade do Estado do Rio de Janeiro, Rio de Janeiro, Brasil \\ Keyth de Oliveira Vianna - Universidade do Estado do Rio de Janeiro, Rio de Janeiro, Brasil \\ Lucimar da Costa Torres Electo - Universidade do Estado do Rio de Janeiro, Rio de Janeiro, Brasil \\ Layse Costa Pinheiro - Universidade do Estado do Rio de Janeiro, Rio de Janeiro, Brasil
}

\begin{abstract}
Resumo
O estudo teve como objetivo investigar a validade convergente do Inventário de Empatia. A amostra foi composta por 230 indivíduos adultos, de ambos os sexos, que responderam ao Inventário de Empatia (IE) e à Escala Multidimensional de Reatividade Interpessoal (EMRI). Correlações positivas e significativas fortes ou moderadas foram obtidas entre os fatores cognitivos do IE (Tomada de Perspectiva e Flexibilidade Interpessoal) e o fator cognitivo da EMRI. No entanto, os fatores afetivos do IE (Altruísmo e Sensibilidade Afetiva) apresentaram corelações positivas e significativas, porém baixas, ou correlações não-significativas, com os fatores afetivos da Escala EMRI. Os achados fornecem, portanto, evidências iniciais acerca da validade convergente dos fatores cognitivos do IE e aprimoram suas qualidades psicométricas no que diz respeito à avaliação da habilidade empática.

Palavras-chave: Empatia, Autorrelato, Testes psicológicos.
\end{abstract}

\section{Convergent validity of the Empathy Inventory (EI)}

\begin{abstract}
The study aimed at investigating the convergent validity of the Empathy Inventory. The sample was composed by 230 adult individuals from both sexes who answered to the Empathy Inventory (EI) and to the Multidimensional Scale of Interpersonal Reactivity (MSIR). Strong or moderate positive and significant correlations were obtained between the EI's cognitive factors (Perspective Taking and Interpersonal Flexibility) and the MSIR's cognitive factor. Therefore, the EI's affective dimensions (Altruism and Affective Sensibility) showed low positive significant correlations or no correlations with the MSIR's affective factors. These findings provide initial evidences about the convergent validity of EI and refine its psychometric qualities as a measure of assessment of empathic skills.

Keywords: Empathy, Self-report, Psychological tests.
\end{abstract}

Validez convergente del Inventario de Empatía (IE)

\begin{abstract}
Resumen
El propósito del estudio fue investigar la validez convergente del Inventario de Empatía. La muestra fue compuesta por 230 individuos adultos de ambos sexos, que respondieron al Inventario de Empatía (IE) y a la Escala Multidimensional de Reactividad Interpersonal (EMRI). Correlaciones positivas y significativas fuertes o moderadas fueran obtenidas entre los factores cognitivos del IE (Toma de Perspectiva y Flexibilidad Interpersonal) y el factor cognitivo de la EMRI. No obstante, los factores afectivos del IE (Altruismo y Sensibilidad Afectiva) presentaron correlaciones positivas y significativas, pero bajas o no significativas con los factores afectivos de la EMRI. Los resultados proporcionan evidencias iniciales acerca de la validez convergente de los factores cognitivos del IE y perfeccionan sus cualidades psicométricas por lo que respecta a la evaluación de la habilidad empática.

Palabras clave: Empatía, Autoinforme, Pruebas psicológicas.
\end{abstract}

A empatia consiste em uma habilidade de comunicação primordial para a qualidade das relações sociais e para a saúde mental. Nesse sentido, sua manifestação tem sido relacionada à formação de vínculos afetivos seguros (Preston \& de Waal, 2002), à promoção do bem-estar pessoal (Canale \& Beckley, 1999; Moll \& cols., 2006), à satisfação conjugal (Cramer \& Jowett, 2010; Oliveira, Falcone \& Ribas Jr., 2009; Sardinha, Falcone \& Ferreira, 2009) e, na relação terapêutica, a melhores efeitos no tratamento (Falcone, Gil \& Ferreira, 2007).
Outrossim, deficiências em experimentar e expressar empatia relacionam-se à agressividade na infância (Pavarino, Del Prette \& Del Prette, 2005), a conflitos conjugais (Epstein \& Schlesinger, 2004), a transtornos do espectro autista (Decety \& Jackson, 2004), à esquizofrenia (Luz \& Bussab, 2009), a transtornos de personalidade (Beck, Freeman \& Davis, 2005; Decety \& Jackson, 2004), e à ansiedade elevada e estilo cognitivo disfuncional (Davis \& Krauss, 1997).

A empatia é compreendida como um fenômeno multidimensional complexo, por envolver 
componentes cognitivos, afetivos e comportamentais. O componente cognitivo refere-se à capacidade de inferir sentimentos e pensamentos de alguém em um determinado contexto, sendo também conhecido como tomada de perspectiva (Falcone \& cols., 2008). O interesse genuíno em compartilhar sentimentos, experimentar compaixão, preocupação ou consideração pelo estado de outra pessoa constitui o componente afetivo da empatia (Falcone, Gil \& Ferreira, 2007). Já o componente comportamental é caracterizado pelas expressões, verbais e não-verbais, de entendimento do estado da pessoa-alvo (Ickes, Marangoni \& Garcia, 1997).

Outra subclasse de fenômenos que compartilham dos mesmos mecanismos subjacentes à empatia é a angústia pessoal, que corresponde a uma experiência vicária experimentada como um desconforto ao testemunhar o sofrimento de alguém, motivando o distanciamento. Ela reflete, entretanto, um sentimento auto-orientado, em vez de orientado para o outro (Batson, 2009). Embora todas as manifestações emocionais (contágio, compaixão, angústia pessoal etc.) estejam envolvidas na experiência da empatia (Preston \& de Waal, 2002), a tomada de perspectiva parece ser um elemento-chave que regula essas emoções de modo a conduzir a uma ação em prol do outro, superando a angústia pessoal e favorecendo a ajuda (Decety \& Jackson, 2004; Decety \& Lamm, 2009).

A empatia motiva ação altruísta e comportamentos de ajuda (Batson, 1997), contribuindo para a elevação da autoestima (Canale \& Beckley, 1999), além de ativar no cérebro o mesmo sistema de gratificação decorrente do ganho de uma recompensa (Moll \& cols., 2006). Por outro lado, o altruísmo excessivo tem sido relacionado a autossacrifício, o qual pode gerar mágoa e depressão (Young, Klosko \& Weishaar, 2003). Assim, é possível que exista um nível ideal de altruísmo dentro de um continum, acima do qual os seus efeitos passam a ser nocivos para o bemestar do indivíduo, comprometendo a satisfação pessoal.

A empatia é influenciada pelas relações de apego em fases precoces do desenvolvimento, as quais aumentam a autorregulação e a competência emocional da criança (Preston \& de Waal, 2002). Nesse sentido, estudos apontam a empatia como relacionada diretamente a estilos seguros de apego e inversamente a apego inseguro (Britton \& Fuendeling, 2005; Burnette, Davis, Green, Worthington \& Bradfield, 2009; Mikulincer, Shaver, Gillath \& Nitzberg, 2005), sendo essas relações atribuídas principalmente ao componente afetivo (Britton \& Fuendeling, 2005; Mikulincer e cols., 2005) ou ao componente cognitivo (Mikulincer, 1998) da empatia.
Em síntese, a capacidade para experimentar e expressar empatia é crucial para a construção e manutenção de relações interpessoais gratificantes, bem como de vínculos afetivos seguros. Ao tomar a perspectiva de outra pessoa, considerando os sentimentos desta, o indivíduo torna-se mais apto a inibir padrões egocêntricos de angústia pessoal ou a manejar os sentimentos de raiva de forma mais adaptativa, facilitando a comunicação e o comportamento de ajuda.

A importante influência que a empatia exerce sobre o bem-estar individual e social tem ensejado o desenvolvimento ou adaptação de diferentes instrumentos de autorrelato destinados a avaliar tal construto, tanto no Brasil (Falcone \& cols., 2008; Koller, Camino \& Ribeiro, 2001) quanto no exterior (Davis, 1980; Hogan, 1969; Mehrabian \& Epstein, 1972). O Inventário de Empatia (IE), de autoria de Falcone e cols. (2008), caracteriza-se como um instrumento brasileiro de autorrelato que avalia quatro dimensões da empatia: Tomada de Perspectiva (TP) refere-se à capacidade de entender a perspectiva e os sentimentos da outra pessoa, mesmo em situações que envolvam conflito de interesses; Flexibilidade Interpessoal (FI) expressa a capacidade para tolerar comportamentos, atitudes e pensamentos muito diferentes ou provocadores de frustração; Altruísmo (Al) reflete a capacidade para sacrificar temporariamente os próprios interesses com a finalidade de beneficiar ou ajudar alguém; Sensibilidade Afetiva (SA) indica sentimentos de compaixão, interesse ou consideração pelo estado do outro. Os dois primeiros fatores do IE representam dimensões cognitivas da empatia e os dois últimos, dimensões afetivas.

Os estudos iniciais realizados com o IE, baseados em análises fatoriais exploratórias e cálculos de consistência interna, evidenciaram que o referido instrumento apresentou adequada estrutura interna e bons índices de precisão. Tais indicadores demonstraram, assim, a pertinência de se realizarem mais estudos com o instrumento, capazes de contribuir para o aprimoramento de suas qualidades psicométricas. Fundamentando-se em tais considerações, o presente estudo teve como objetivo reunir evidências adicionais acerca da validade de construto do IE, mediante a verificação de sua validade convergente. Para tanto, foram investigadas as correlações desse instrumento com outra medida brasileira de empatia. Uma vez que ambos os instrumentos destinam-se a medir a empatia, é de se esperar que seus diferentes fatores apresentem-se significativamente correlacionados. 


\section{Participantes}

\section{Método}

Participaram deste estudo 230 indivíduos adultos, de ambos os sexos, sendo 43,9\% do sexo masculino. As idades variaram de 18 a 63 anos, com média de 29, 23 e desvio padrão de 11,84 anos. Os níveis de escolaridade incluíram: fundamental completo $(2,6 \%)$, médio incompleto $(3,5 \%)$, médio completo $(21,7 \%)$, graduação incompleta $(50,4 \%)$, superior completo $(11,7 \%)$ e pós-graduação $(10,1 \%)$. Quanto ao estado civil, $70,9 \%$ dos participantes eram solteiros, $24,7 \%$, casados, e 4,4\%, separados.

\section{Instrumentos}

Os instrumentos utilizados no estudo foram o Inventário de Empatia (IE) e a Escala Multidimensional de Reatividade Interpessoal (EMRI). O IE consiste em um instrumento de autorrelato composto de 40 itens, os quais devem ser respondidos em escalas de cinco pontos, variando de 1 (nunca) a 5 (sempre), segundo a frequência com que cada uma das situações sociais descritas aplica-se ao respondente. Tais itens se distribuem por quatro subescalas: Tomada de Perspectiva (12 itens); Flexibilidade Interpessoal (10 itens); Altruísmo ( 9 itens); Sensibilidade Afetiva (9 itens). $\mathrm{Na}$ avaliação dos resultados da escala, os itens negativos (17 itens) devem ser invertidos para que, em seguida, sejam calculadas as médias das pontuações obtidas nos itens pertencentes a cada fator. O instrumento é corrigido na direção da empatia e, assim, quanto maior o resultado maior o grau do fator considerado.

A EMRI é uma medida de empatia desenvolvida por Davis (1980) e adaptada e validada para a população brasileira por Koller e colaboradores (2001). A versão brasileira apresenta 21 itens, com respostas numeradas de 1 (não me descreve bem) a 5 pontos (descreve-me muito bem). Tais itens se distribuem em três subescalas, cada uma com sete itens: Consideração Empática (contém componentes afetivos da empatia e reflete a preocupação para com outras pessoas);
Tomada de Perspectiva (contém componentes cognitivos da empatia e indica a tendência para adotar o ponto de vista psicológico do outro, antecipando seus comportamentos e reações); Mal-Estar Pessoal (caracteriza-se por ansiedade e preocupação autoorientada ao perceber os outros em aflição). Para a correção da escala, deve-se inicialmente inverter os itens negativos (6 itens) e, em seguida, calcular as médias das pontuações obtidas nos itens pertencentes a cada fator. $O$ instrumento também é corrigido no sentido da empatia, de forma que quanto maior o resultado, maior o grau do fator considerado. O questionário de coleta de dados incluiu ainda o Termo de Consentimento Livre e Esclarecido, com o propósito de prestar esclarecimentos sobre a pesquisa e sobre os direitos do participante, e uma ficha utilizada para a obtenção dos dados sociodemográficos da amostra.

\section{Procedimento}

A pesquisa teve início após a aprovação do projeto de pesquisa pelo Comitê de Ética da instituição do autor principal. Os participantes foram recrutados com base na rede social dos pesquisadores $\mathrm{e}$ responderam individualmente aos instrumentos, tendo a opção de completarem as medidas na hora ou levarem-nas para completar em casa e devolverem em data combinada com o pesquisador.

\section{Resultados}

Os dados obtidos foram transpostos para uma planilha do SPSS (Statistical Package for Social Sciences) e analisados estatisticamente por meio do mesmo programa. Para avaliar a validade convergente do IE, foram calculados os coeficientes de correlação de Pearson entre os quatro fatores do IE e os três fatores da EMRI. A Tabela 1 indica os valores dos coeficientes de correlação obtidos, bem como seus níveis de significância.

Tabela 1. Correlações entre os Fatores do IE e da EMRI

\begin{tabular}{lccc}
\hline \multirow{2}{*}{ IE } & \multicolumn{3}{c}{ EMRI } \\
\cline { 2 - 4 } & Consideração Empática & Tomada de Perspectiva & Mal-Estar Pessoal \\
\hline Tomada de Perspectiva & $0,26^{* *}$ & $0,61^{* *}$ & $-0,19^{* *}$ \\
Flexibilidade Interpessoal & 0,06 & $0,31^{* *}$ & $-0,17^{*}$ \\
Altruísmo & 0,09 & 0,02 & $-0,01$ \\
Sensibilidade Afetiva & $0,16^{*}$ & $0,19^{* *}$ & $-0,05$ \\
\hline
\end{tabular}

${ }^{*} \mathrm{p}<0,05 /{ }^{* *} \mathrm{p}<0,01$

A subescala de Tomada de Perspectiva do IE apresentou uma correlação positiva e significativa com a subescala de Tomada de Perspectiva da EMRI $(r=0,61 ; \quad p<0,01), \quad$ podendo tal correlação ser classificada como forte, segundo os critérios de Miles e Shevlin (2001). Tal resultado encontra-se de acordo 
com o esperado, na medida em que ambas as subescalas estão avaliando um mesmo fator, qual seja a tomada de perspectiva. A subescala de Tomada de Perspectiva do IE também se correlacionou positiva e significativamente com a escala de Consideração Empática da EMRI $(r=0,26 ; p<0,01)$. No entanto, a correlação entre essas escalas, apesar de significativa, foi baixa (Miles \& Shevlin, 2001). Esse resultado seria também de se esperar, já que a tomada de perspectiva consiste em uma dimensão cognitiva da empatia, enquanto a consideração empática caracteriza-se como uma dimensão afetiva do construto.

A subescala de Flexibilidade Interpessoal do IE correlacionou-se positiva e significativamente com a subescala de Tomada de Perspectiva da EMRI $(r=0,31$; $p<0,01)$, mas de forma apenas moderada (Miles \& Shevlin, 2001). Esse resultado também era previsto, pois ambos os fatores são de natureza cognitiva. Já o Fator Altruísmo do IE não apresentou correlações significativas com nenhum dos fatores da EMRI, o que também se encontra de acordo com o esperado, pois nenhum dos fatores da EMRI avalia o comportamento altruísta.

A subescala de Sensibilidade Afetiva do IE, por fim, obteve uma correlação positiva e significativa com a subescala de Tomada de Perspectiva $(r=0,19 ; p<0,01)$ da EMRI, mas baixa, o que se mostra coerente com o fato de a sensibilidade afetiva se constituir em uma dimensão afetiva da empatia, enquanto a tomada de perspectiva se refere a uma dimensão cognitiva do construto. Contrariando, porém, o que seria de se esperar, a correlação da subescala de Sensibilidade Afetiva do IE com a subescala de Consideração Empática $(r=0,16 ; p<0,05)$ da EMRI foi, também, baixa, apesar de positiva e significativa, o que não se coaduna com a natureza dessas duas facetas, na medida em que ambas consistem em dimensões afetivas da empatia associadas à preocupação e cuidado com o estado do outro.

\section{Discussão}

Foi objetivo geral do presente estudo, reunir evidências adicionais acerca da validade de construto do Inventário de Empatia (IE), mediante a verificação de sua validade convergente. O IE compõe-se de dois fatores de natureza cognitiva (Tomada de Perspectiva e Flexibilidade Interpessoal) e dois de natureza afetiva (Altruísmo e Sensibilidade Afetiva).

As duas subescalas cognitivas do IE correlacionaram-se positiva e significativamente com a única subescala de natureza cognitiva da EMRI (Tomada de Perspectiva). Contudo, a correlação entre as subescalas de Tomada de Perspectiva de ambos os inventários foi forte, o que pode ser visto como uma evidência de que ambas as subescalas estão avaliando uma mesma dimensão da empatia associada à capacidade de se adotar o que ponto de vista do outro (Falcone \& cols., 2008). O outro fator cognitivo do IE (Flexibilidade Interpessoal) correlacionou-se apenas de forma moderada com a subescala de Tomada de Pesrpectiva da EMRI, o pode ser considerado uma demonstração de que, embora ambas as dimensões sejam de natureza cognitiva, elas provavelmente avaliam nuances distintas do componente cognitivo da empatia. De todo modo, os resultados ora obtidos podem ser considerados como evidências iniciais acerca da validade convergente dos fatores cognitivos do IE, em especial a Tomada de Perspectiva.

No que diz respeito aos fatores afetivos do IE, foi possível observar que Altruísmo não se correlacionou com nenhum dos dois fatores afetivos da EMRI. Esse resultado encontra-se, no entanto, dentro do previsto, em virtude de o altruísmo refletir a capacidade de se sacrificar temporariamente em prol do outro (Falcone \& cols., 2008), enquanto os dois fatores afetivos da EMRI - Consideração Empática e Mal-Estar Pessoal associam-se, respectivamente, à preocupação com o outro e à ansiedade decorrente da percepção do outro em aflição (Koller \& cols., 2001). Parece, assim, que o altruísmo envolve não apenas um componente afetivo, mas também um componente comportamental, o que justificaria o fato de ele não ter se correlacionado com os dois fatores puramente afetivos da EMRI. Outrossim, o resultado ora obtido pode ser visto como uma evidência inicial acerca da validade divergente do fator Altruísmo.

Atendo-se por fim, à subescala de Sensibilidade Afetiva do IE, foi possível observar que ela apresentou uma correlação positiva e significativa somente com um dos fatores afetivos da EMRI (Consideração Empática) e, mesmo assim, contrariando o que seria esperado, tal correlação foi fraca. Uma possível explicação para tal resultado pode ser a de que apesar de ambas as dimensões serem de natureza afetiva, a consideração empática reflete uma preocupação mais generalizada com outras pessoas (Falcone \& cols., 2008), enquanto a sensibilidade afetiva é mais focada na compaixão, interesse ou consideração pelo estado do outro (Koller \& cols., 2001). Ainda assim, os atuais achados denotam não ter sido possível reunir evidências consistentes acerca da validade convergente do fator Sensibilidade Afetiva do IE.

\section{Considerações finais}

Os resultados obtidos permitem a conclusão de que foram obtidas evidências iniciais sobre a validade 
convergente dos fatores cognitivos do IE, em especial a Tomada de Perspectiva. Ademais, os achados acerca da subescala de Altruísmo ofereceram indicações preliminares sobre sua validade divergente, embora esse não fosse um objetivo da atual investigação. Não foi possível, no entanto, obter conclusões mais consistentes acerca da validade convergente da subescala de Sensibilidade Afetiva, em virtude das baixas correlações observadas entre essa escala e os fatores da EMRI a ela associados conceitualmente, em que pese $\mathrm{o}$ fato de elas terem se mostrado significativas.

Atendo-se a uma agenda futura de pesquisas destinadas a aprimorar as características psicométricas do Inventário de Empatia, sugere-se que novos estudos investiguem a validade concorrente e preditiva do instrumento. Nesse sentido, investigações direcionadas à verificação da capacidade do IE em diferenciar grupos clínicos e não-clínicos e de predizer determinados tipos de comportamentos futuros, como, por exemplo, o desempenho em atividades profissionais que exijam competências interpessoais, poderiam contribuir ainda mais para o aprimoramento da escala. Outrossim, pesquisas voltadas à verificação de sua validade divergente também poderão oferecer contribuições valiosas.

Cumpre ressaltar, por fim, as limitações do presente estudo. Assim é que a correlação apenas moderada obtida entre a subescala de Flexibilidade Interpessoal do IE e a subescala de Tomada de Perspectiva da EMRI denota que as evidências iniciais acerca da validade convergente dessa subescala do IE devem ser consideradas com cautela. Por outro lado, o fato de a coleta de dados não ter sido padronizada, isto é, de os questionários terem sido aplicados e entregues logo após seu preenchimento ou devolvidos posteriormente, pode ter interferido nos resultados presentemente observados.

De todo modo, as evidências aqui obtidas contribuíram para aprimorar as qualidades psicométricas do Inventário de Empatia, no que tange à sua validade convergente. Torna-se, porém, necessário que investigações futuras permitam $\mathrm{O}$ alargamento dos contextos e propósitos para os quais o IE poderá ser adotado.

\section{Referências}

Batson, C. D. (1997). Self-other merging and the empathy - altruism hypothesis: reply to Neuberg et al. Journal of Personality and Social Psychology, 73, 517-522.

Batson, C. D. (2009). These things called empathy: eight related but distinct phenomena. Em J.
Decety, \& W. Ickes (Orgs.). The social neuroscience of empathy (pp. 3-15). Massachusetts: Bradford Book.

Beck, A. T., Freeman, A. \& Davis, D. D. (2005). Terapia cognitiva dos transtornos de personalidade. $2^{\mathrm{a}}$ ed. Porto Alegre: Artmed.

Britton, C. P., \& Fuendeling, J. M. (2005). The relations among varieties of adult attachment and the components of empathy. The Journal of Social Psychology, 145, 519-530.

Burnette, J. L., Davis, D. E., Green, J. D., Worthington, E. L., Jr. \& Bradfield, E. (2009). Insecure attachment and depressive symptoms: the mediating role of rumination, empathy, and forgiveness. Personality and Individual Differences, 46, 276-280.

Canale, J. R. \& Beckley, S. R. (1999). Promoting altruism in troubled youth: considerations and suggestions. North American Journal of Psychology, 1, 95-102.

Cramer, D. \& Jowett, S. (2010). Perceived empathy, accurate empathy and relationship satisfaction in heterosexual couples. Journal of Social and Personal Relationships, 27, 327-349.

Davis, M. H. (1980). A multidimensional approach to individual differences in empathy. Catalog of Selected Documents in Psychology, 10, 85.

Davis, M. H. \& Kraus, L. A. (1997). Personality and empathic accuracy. Em W. Ickes (Org.). Empathic accuracy (pp. 144-168). New York: Guilford.

Decety, J. \& Jackson, P. L. (2004). The functional architecture of human empathy. Behavioral and Cognitive Neuroscience Reviews, 3, 71-100.

Decety, J. \& Lamm, C. (2009). Empathy versus personal distress: recent evidence from social neuroscience. Em J. Decety, \& W. Ickes (Orgs.). The social neuroscience of empatby (pp. 199-213). Massachusetts: Bradford Book.

Epstein, N. B. \& Schlesinger, S. E. (2004). Casais em crise. Em F. M. Dattilio \& A. Freeman (Orgs.). Estratégias cognitivo-comportamentais de intervenção em situações de crise (pp. 243-263). $2^{\mathrm{a}}$ ed. Porto Alegre: Artmed.

Falcone, E. M. O., Gil, D. B. \& Ferreira, M. C. (2007). Um estudo comparativo da freqüência de verbalização empática entre psicoterapeutas de diferentes abordagens teóricas. Estudos de Psicologia, 24, 451-461.

Falcone, E. M. O., Ferreira, M. C., Luz, R. C. M., Fernandes, C. S., Faria, C. A., D'Augustin, J. F., Sardinha, A. \& Pinho, V. D. (2008). Inventário de Empatia (I.E.): desenvolvimento e validação de uma medida brasileira. Avaliação Psicológica, 7, 321334. 
Hogan, R. (1969). Development of an empathy scale. Journal of Consulting and Clinical Psychology, 33, 307316.

Ickes, W., Marangoni, C. \& García, S. (1997). Studying empathic accuracy in a clinically relevant context. Em W. Ickes (Org). Empathic accuracy (pp. 282310). Nova Iorque: Guilford.

Koller, S. H., Camino, C., \& Ribeiro, J. (2001). Adaptação e validação interna de duas escalas de empatia para uso no Brasil. Estudos de Psicologia, 18, 43-53.

Luz, F. \& Bussab, V. S. R. (2009). Psicopatologia evolucionista. Em E. Otta, \& M. E. Yamamoto (Orgs.). Psicologia evolucionista (pp. 163-175). Rio de Janeiro: Guanabara Koogan.

Mehrabian, A., \& Epstein, N. (1972). A measure of emotional empathy. Journal of Personality, 40, 525543.

Mikulincer, M. (1998). Adult attachment style individual differences in functional versus dysfunctional experiences of anger. Journal of Personality and Social Psychology, 74, 513-524.

Mikulincer, M., Shaver, R. P., Gillath, O. \& Nitzberg, R. A. (2005). Attachment, caregiving, and altruism: boosting attachment security increases compassion and helping. Journal of Personality and Social Pyichology, 89, 817-839.

Miles, J. N. V. \& Shevlin, M. E. (2001). Applying regression and correlation: a guide for students and researchers. Londres: Sage.
Moll, J., Krueger, F., Zahn, R., Pardini, M., OliveiraSouza, R. \& Grafman, J. (2006). Human frontomesolimbic networks guide decisions about charitable donation. Proceedings of the National Academy of Sciences, 103, 15623-15628.

Oliveira, M. G. S., Falcone, E. M. O. \& Ribas Jr., R. C. (2009). A avaliação das relações entre a empatia e a satisfação conjugal: um estudo preliminar. Interação em Psicologia, 13, 287-298.

Pavarino, M. G., Del Prette, A. \& Del Prette, Z. A. P. (2005). O desenvolvimento da empatia como prevenção da agressividade na infância. PSICO, 36, 127-134.

Preston, S. D. \& de Waal, F. B. M. (2002). Empathy: its ultimate and proximate bases. Behavioral and Brain Sciences, 25, 1-72.

Sardinha, A., Falcone, E. M. O. \& Ferreira, M. C. (2009). As relações entre a satisfação conjugal e as habilidades sociais percebidas no cônjuge. Psicologia: Teoria e Pesquisa, 25, 395-402.

Young, J. E., Klosko, J. S., \& Weishaar, M. E. (2003). Schema therapy. A practitioner's guide. Nova Iorque: The Guilford Press.
Recebido em 25/05/2011

Reformulado em 10/01/2013

Aprovado em 14/05/2013 
Sobre as autoras

Eliane Mary de Oliveira Falcone é mestre e doutora em Psicologia, professora adjunta da Universidade do Estado do Rio de Janeiro, docente do Programa de Pós-Graduação em Psicologia Social da UERJ. Ex-Presidente da Federação Brasileira de Terapias Cognitivas (FBTC) no período de 2003 a 2005, é fundadora da Revista Brasileira de Terapias Cognitivas (RBTC), da qual foi Editora responsável até 2009.

Vanessa Dordron de Pinho é doutoranda e mestre em Psicologia Social pelo Programa de Pós-Graduação em Psicologia Social da UERJ, segunda-secretária da Associação de Terapias Cognitivas do Estado do Rio de Janeiro (ATC-Rio) na gestão 2011-2014 e psicóloga da Fundação de Saúde da Prefeitura de Angra dos Reis.

Maria Cristina Ferreira é mestre e doutora em Psicologia, professora titular e coordenadora do Programa de PósGraduação em Psicologia da Universidade Salgado de Oliveira. Secretária da ANPEPP no período de 2008-2010 e vice-presidente no período de 2010-2012, é representante da América Latina no Comitê Executivo da International Association of Cross-Cultural Psychology.

Conceição dos Santos Fernandes é mestre pelo Programa de Pós-Graduação em Psicologia Social da UERJ, graduada no curso de Psicologia pela Universidade do Estado do Rio de Janeiro.

Juliana Furtado D’Augustin é doutoranda e mestre em Psicologia Social pela Universidade do Estado do Rio de Janeiro, psicóloga do Núcleo de Assistência e Pesquisa em Transtornos Alimentares da Policlínica Piquet Carneiro/UERJ, e membro da diretoria da Associação de Terapias Cognitivas do Estado do Rio de Janeiro - ATCRio (Gestão 2011-2013).

Stèphanie Krieger é graduanda de Psicologia da UERJ. Foi bolsista CNPq (2010-2011) e é FAPERJ (2011-atual) de Iniciação Científica e estagiária em Terapia Cognitivo-Comportamental do Serviço de Psicologia Aplicada - UERJ, do Núcleo de Assistência e Pesquisa em Transtornos Alimentares - Policlínica Piquet Carneiro e do setor de Dependência Química e Outros Transtornos do Impulso - Santa Casa da Misericórdia do Rio de Janeiro.

Monique Gomes Plácido é graduanda em psicologia pela UERJ, membro do grupo de pesquisa em Relações Interpessoais e Habilidades Sociais (2009 - atual), bolsista de Iniciação Científica da Fundação de Amparo à Pesquisa do Estado do Rio de Janeiro - FAPERJ (2012-atual) e estagiária em Terapia Cognitivo-Comportamental do SPA - UERJ e do Núcleo de Assistência e Pesquisa em Transtornos Alimentares - Policlínica Piquet Carneiro.

Keyth de Oliveira Vianna é graduanda de psicologia da UERJ, membro do grupo de pesquisa em Relações Interpessoais e Habilidades Sociais (2009-2011) e estagiária em Gestalterapia do SPA-UERJ.

Lucimar da Costa Torres Electo é graduanda em Psicologia pela UERJ, membro do grupo de pesquisa em Relações Interpessoais e Habilidades Sociais (2009 - atual), bolsista FAPERJ (2011-2012) e estagiária em Terapia Cognitivo-Comportamental do SPA-UERJ.

Layse Costa Pinheiro é graduanda de Psicologia da UERJ, bolsista CNPq (2008/2009 e 2010/2011), PIBIC/UERJ (2009/2010) de Iniciação Científica e FAPERJ (2012) de Extensão. Estagiária em Terapia CognitivoComportamental no SPA-UERJ (2010/2011), é coordenadora do Núcleo de Projetos da Empresa Júnior de Psicologia da UERJ (2011/2012) e monitora do Curso de Extensão "Psicologia nas Organizações: Prática em Gestão de Pessoas”, CEPUERJ 2012.

Contato com as autoras

Rua Visconde Silva, 154, ap. 404 - CEP 22271-044 - Humaitá - Rio de Janeiro-RJ, Brasil. 